\title{
A Concise Representation of Association Rules Using Minimal Predictive Rules
}

\author{
Iyad Batal and Milos Hauskrecht \\ Department of Computer Science \\ University of Pittsburgh \\ \{iyad,milos\}acs.pitt.edu
}

\begin{abstract}
Association rule mining is an important branch of data mining research that aims to extract important relations from data. In this paper, we develop a new framework for mining association rules based on minimal predictive rules (MPR). Our objective is to minimize the number of rules in order to reduce the information overhead, while preserving and concisely describing the important underlying patterns. We develop an algorithm to efficiently mine these MPRs. Our experiments on several synthetic and UCI datasets demonstrate the advantage of our framework by returning smaller and more concise rule sets than the other existing association rule mining methods.
\end{abstract}

\section{Introduction}

The huge amounts of data collected today provide us with an opportunity to better understand the behavior and structure of many natural and man-made systems. However, the understanding of these systems may not be possible without automated tools that enable us to extract the important patterns in the data and present them in a concise and easy to understand form.

Rule induction methods represent a very important class of knowledge discovery tools. The advantage of these methods is that they represent the knowledge in terms of if-then rules that are easy to interpret by humans. This can facilitate the process of discovery and utilization of new practical findings. As an example, consider a knowledge discovery problem in medicine. Assume a rule mining algorithm identifies a subpopulation of patients that respond better to a certain treatment than the rest of the patients. If the rule clearly and concisely defines this subpopulation, it can speed up the validation process of this finding and its future utilization in patient-management.

Association rule mining is a very popular data mining technique to extract rules from the data. The original framework [1] has been extended to mine patterns from various domains [28,4 16]. The key strength of association rule mining is that it searches the space of rules completely by examining all patterns that occur frequently in the data. However, the main disadvantage is that the number of association rules it finds is often very large. Moreover, many rules are redundant because they can be naturally explained by other rules. This may hinder the discovery process and the interpretability of the results. The objective of this work is to filter out these redundant rules and provide the user with a small set of rules that are sufficient to capture the essential underlying structure of the data. 
In this work, we focus on the problem of mining association rules that target a specific class variable of interest. We call these rules the class association rules. To achieve our goal, we first introduce the concept of the minimal predictive rules (MPR) set that assures a good coverage of the important patterns with very small number of rules. After that, we propose an algorithm for mining these rules. Briefly, our method builds the MPR set by examining more general rules first and gradually testing and adding more specific rules to the set. The algorithm relies on a statistical significance test to ensure that every rule in the result is significantly better predictor than any of its generalizations.

\section{Methodology}

In this section, we first define basic terminology used throughout the paper. After that, we present an example illustrating the challenges of rule mining and the limitations of existing methods. Next, we propose the minimal predictive rules (MPR) framework to address them. Finally, we present an algorithm for mining the MPRs.

\subsection{Definitions}

Our work focuses on mining relational databases, where each record is described by a fixed number of attributes. We assume that all attributes have discrete values (numeric attributes must be discretized [14]). We call an attribute value pair an item and a conjunction of items a pattern (sometimes patterns are also called itemsets). If a pattern contains $k$ items, we call it a k-pattern (an item is a 1-pattern). We say that pattern $P^{\prime}$ is a subpattern of pattern $P$ if $P^{\prime} \subset P\left(P\right.$ is a superpattern of $\left.P^{\prime}\right)$. A rule is defined as $R: A \Rightarrow c$, where $A$ is a pattern and $c$ is the class label that $R$ predicts. We say that rule $R^{\prime}: A^{\prime} \Rightarrow c^{\prime}$ is a subrule of rule $R: A \Rightarrow c$ if $c^{\prime}=c$ and $A^{\prime} \subset A$.

A pattern $P$ can be viewed as defining a subpopulation of the instances (records) that satisfy $P$. Hence, we sometimes refer to pattern $P$ as group $P$. If $P^{\prime}$ is a subpattern of $P$, then $P^{\prime}$ is a supergroup of $P$. Note that the empty pattern $\Phi$ defines the entire population. The support of pattern $P$, denoted as $\sup (P)$, is the ratio of the number of records that contain $P$ to the total number of records: $\sup (P) \approx \operatorname{Pr}(P)$. The confidence of rule $R: A \Rightarrow c$ is the posterior probability of class $c$ in group $A$ : $\operatorname{conf}(R)=\sup (A \cup$ $c) / \sup (A) \approx \operatorname{Pr}(c \mid A)$. Note that confidence of the empty rule is the prior probability of the class: $\operatorname{conf}(\Phi \Rightarrow c) \approx \operatorname{Pr}(c)$.

\subsection{Example}

Assume our objective is to identify populations which are at high risk of developing coronary heart disease (CHD). Assume that our dataset contains 200 instances and that the $\mathrm{CHD}$ prior is $\operatorname{Pr}(\mathrm{CHD})=30 \%$. We want to evaluate the following 3 rules:

$\mathrm{R} 1$ : Family history=yes $\Rightarrow \mathrm{CHD}$

$$
\text { [sup }=50 \%, \text { conf }=60 \%]
$$

R2: Family history $=$ yes $\wedge$ Race $=$ Caucasian $\Rightarrow$ CHD

$$
\text { [ } \sup =20 \%, \operatorname{conf}=55 \% \text { ] }
$$

R3: Family history=yes $\wedge$ Race $=$ African American $\Rightarrow \mathrm{CHD}$

$$
[\text { sup }=20 \%, \text { conf }=65 \%]
$$


From the above rules, we can see that a positive family history is probably an important risk factor for CHD because the confidence of R1 (60\%) is two times higher than CHD prior $(30 \%)$. However, the problem is that we expect many rules that contain a positive family history in their antecedents to have a high confidence as well. So how can we know which of these rules are truly important for describing the CHD condition?

The original association rules framework [1] outputs all the frequent rules that have a higher confidence than a minimum confidence threshold (min_conf). For instance, if we set $\min \_$conf $=50 \%$, all of three rules will be returned to the user.

In order to filter out some of the uninteresting associations, the original supportconfidence framework is sometimes augmented with a correlation measure. Commonly, a $\chi^{2}$ test is used to assure that there is a significant positive correlation between the condition of the rule and its consequent [8|24|21|18]. However, because the posteriors of all three rules are much higher than the prior, we expect all of them to be statistically significant! Moreover, these rules will be considered interesting using most existing interestingness measures [15]. The main problem with this approach is that it evaluates each rule individually without considering the relations between the rules. For example, if we are given rule R2 by itself, we may think it is an important rule. However, by looking at all three rules, we can see that $\mathrm{R} 2$ should not be reported because it is more specific than R1 (applies to a smaller population) and has a lower confidence.

To filter out such redundant rules, [6] defined the confidence improvement constraint:

$$
\operatorname{imp}(A \Rightarrow c)=\operatorname{conf}(A \Rightarrow c)-\max _{A^{\prime} \subset A}\left\{\operatorname{conf}\left(A^{\prime} \Rightarrow c\right)\right\}>\text { min_imp }
$$

In practice, it is not clear how to specify this min_imp parameter. So the common convention is to set to zero ([1817]). This means that we only report the rules that have a higher confidence than all of their subrules. If we applied the confidence improvement constraint to our working example, rule R 2 will be removed and rule R3 will be retained. However, R3 may also be unimportant and its observed improvement in the confidence can be due to chance rather than actual causality. In fact, there is a high chance that a refinement of a rule, even by adding random items, leads to a better confidence. We will see later in the analysis in section 2.4 and in the experimental evaluation that the confidence improvement constraint can still output many spurious rules. So should we report rule R3? To answer this question, we define the minimal predictive rules concept.

\subsection{Minimal Predictive Rules (MPR)}

Definition 1. A rule $R: A \Rightarrow c$ is a minimal predictive rule (MPR) if and only if $\mathrm{R}$ predicts class c significantly better than all its sub-rules.

This definition implies that every item in the condition $(A)$ is an important contributor to the predictive ability of the rule. We call these rules minimal because removing any non-empty combination of items from the condition would cause a significant drop in the predictability of the rule. An MPR can be viewed as defining a "surprising" subpopulation, where the posterior probability of class $c$ in the group $A(\operatorname{Pr}(c \mid A))$ is unexpected and cannot be explained by any convex combinations of $A$ 's subpatterns.

The MPR significance test: In order to check if a rule is significant with respect to its subrules, we use the binomial distribution as follows: Assume we are interested in testing the significance of rule $R: A \Rightarrow c$. Assume that group $A$ contains $N$ instances, out 
of which $N_{c}$ instances belong to class $c$. Assume $P_{c}$ represents the highest confidence achieved by any subrule of $R: P_{c}=\max _{A^{\prime} \subset A} \operatorname{Pr}\left(c \mid A^{\prime}\right)$. The null hypothesis presumes that $N_{c}$ is generated from $N$ according to the binomial distribution with probability $P_{c}$. The alternative hypothesis presumes that the true underlying probability that generated $N_{c}$ is significantly higher than $P_{c}$. Hence, we perform a one sided significance test (we are interested only in increases in the proportion of $c$ ) and calculate a p-value as follows:

$$
p=\operatorname{Pr}_{\text {binomial }}\left(x \geq N_{c} \mid N, P_{c}\right)
$$

If this p-value is significant (smaller than a significance level $\alpha$ ), we conclude that $R$ significantly improves the predictability of $c$ over all its subrules, hence is an MPR.

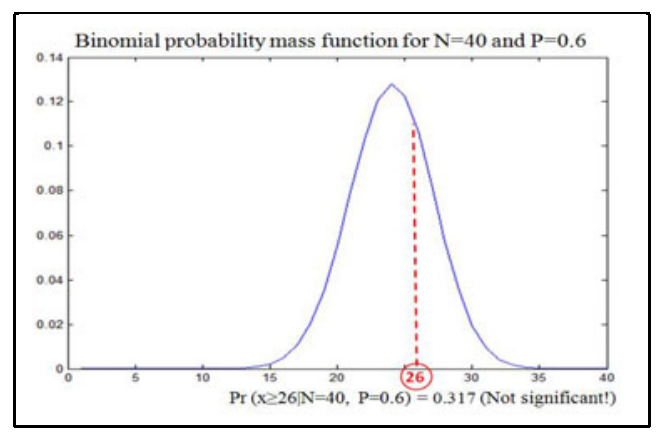

Fig. 1. The MPR significance test for rule R3

Example: Going back to our CHD example, rule R3 covers 40 instances, out of which 26 have CHD. For R3 to be an MPR, it should be significantly more predictive than all its simplifications, including rule R1. By applying the MPR significance test we get: $\operatorname{Pr}_{\text {binomial }}(x \geq 26 \mid 40,0.6)=0.317$. As illustrated in Figure 1, we can see that R3 is not an MPR at significance level $\alpha=0.05$. On the other hand, if we use the same binomial significance test to evaluate each rule individually against the CHD prior (by always setting $P_{c}=\operatorname{Pr}(\mathrm{CHD})$ ), the p-values we get for R1, R2 and R3 are respectively, $5.13 \mathrm{e}-10,8.54 \mathrm{e}-4$ and $5.10 \mathrm{e}-6$, meaning that all three rules are (very) significant!.

\subsection{Spurious Patterns and Redundant Rules}

In this section, we discuss and analyze the serious problem of redundancy in association rules. This problem is due to the manner in which large numbers of spurious rules are formed by adding irrelevant items to the antecedent of other rules. We show the deficiencies of the current approaches to dealing with this problem. Finally, we show how MPR can overcome the problem.

Consider a Bayesian belief network example in Figure 2, where we have a causal relation between variable $X_{1}$ and the class variable $C$. Assume that item $X_{1}=1$ is highly predictive of class $c_{1}$, so that $\operatorname{Pr}\left(C=c_{1} \mid X_{1}=1\right)$ is significantly larger than $\operatorname{Pr}\left(C=c_{1}\right)$. Assume we have another variable $X_{2}$ that is completely independent of $C: X_{2} \Perp C$. If we add any instantiation $v_{2}$ of variable $X_{2}$ to item $X_{1}=1$, the posterior distribution of 


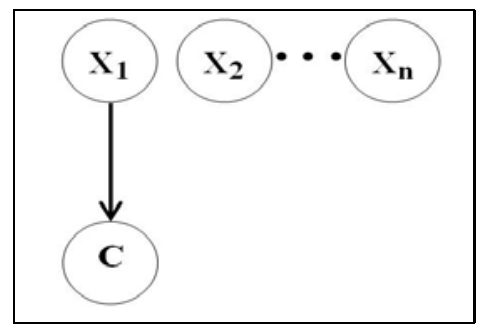

Fig. 2. Illustrating the redundancy problem in association rules

$c_{1}$ is $\operatorname{Pr}\left(C=c_{1} \mid X_{1}=1 \wedge X_{2}=v_{2}\right) \approx \operatorname{Pr}\left(C=c_{1} \mid X_{1}=1\right)$, i.e., $\operatorname{conf}\left(X_{1}=1 \wedge X_{2}=v_{2} \Rightarrow c_{1}\right)$ $\approx \operatorname{conf}\left(X_{1}=1 \Rightarrow c_{1}\right)$.

More generally, if we have many irrelevant variables such that $X_{i} \Perp C: i \in\{2 . . n\}$, the network structure implies that $\operatorname{Pr}\left(C=c_{1} \mid X_{1}=1 \wedge X_{2}=v_{2} \ldots \wedge X_{n}=v_{n}\right) \approx \operatorname{Pr}\left(C=c_{1}\right.$ | $X_{1}=1$ ) for every possible instantiation $X_{i}=v_{i}$. Clearly, the number of such spurious rules can become huge, which can easily overwhelm the user and prevent him from understanding the real patterns and causalities in the data.

Even by requiring the complex rules to have a higher confidence [6 17 18] or lift score [9] than their simplifications, the problem still exists and many of these redundant rules can easily satisfy this constraint. In fact, if $X_{i}$ is a binary variable and $\operatorname{conf}\left(X_{1}=1\right.$ $\left.\wedge X_{i}=0 \Rightarrow c_{1}\right)<\operatorname{conf}\left(X_{1}=1 \Rightarrow c_{1}\right)$, then we know for sure that $\operatorname{conf}\left(X_{1}=1 \wedge X_{i}=1\right.$ $\left.\Rightarrow c_{1}\right)>\operatorname{conf}\left(X_{1}=1 \Rightarrow c_{1}\right)$. The same situation happens if we use the lift score instead of the confidence! Post-pruning the rules individually based on their correlations with the class (e.g. using the $\chi^{2}$ test [8|24|21|18]) or based on their difference from the prior (e.g. using our binomial test) is not going to not help in this case.

Our frameworks tackles this problem because every item in an MPR should significantly contribute to improving the predictability of the rule. This means that if rule $R: X_{q_{1}}=v_{q_{1}} \ldots \wedge X_{q_{k}}=v_{q_{k}} \Rightarrow C=c$ is an MPR, then there should exist a path from each variable $X_{q_{i}}$ to the class $C$ that is not blocked (d-separated) by the other variables in the rule: $X_{q_{i}}$ not $\Perp C \mid\left\{X_{q_{1}}, X_{q_{i-1}}, \ldots, X_{q_{i+1}}, X_{q_{k}}\right\}$. Therefore, redundant rules are likely to be filtered out.

\subsection{The Algorithm}

In this section we explain our algorithm for mining the MPR set. The algorithm is outlined in Figure 3 Briefly, the algorithm explores the space by performing a level wise Apriori-like search. At each level $(l)$, we first remove the candidate $l$-patterns that do not pass the minimum support threshold (line 6). Then we extract all MPRs from these frequent l-patterns (line 7). Finally, we generate the candidates for the next level (line 8).

Extracting MPRs. The process of testing if a rule $P \Rightarrow c$ is an MPR is not trivial because the definition requires us to check the rule against all its proper subrules. This would require to check $2^{l}$ subpatterns if $P$ has length $l$. Our algorithm avoids this inefficiency by caching the statistics needed to perform the check within the (l-1)-subpatterns from the previous level. This part of the algorithm is outlined in Figure 4. 


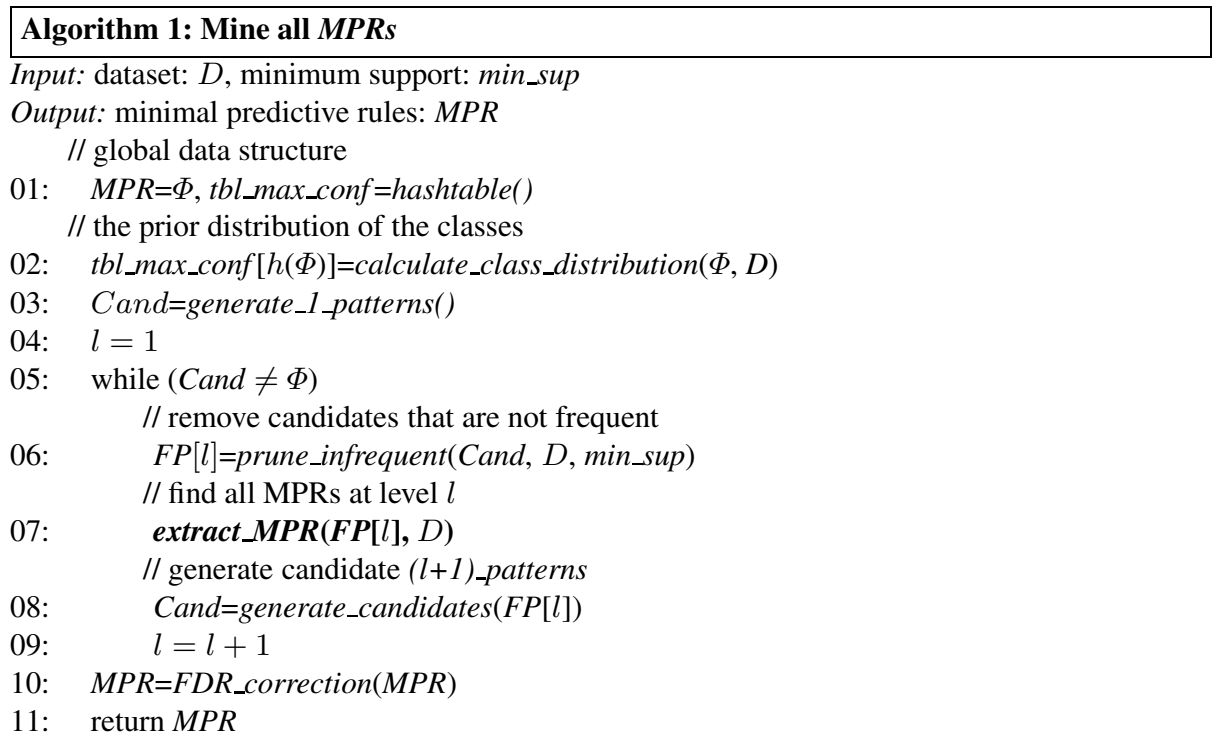

Fig. 3. The algorithm for mining MPRs from a dataset

To explain the method, it is useful to envision the progress of the algorithm as gradually building a lattice structure level by level, starting from the empty pattern $\Phi$. An example lattice is shown in Figure 5. Every frequent $l$-pattern $P$ is a node in the lattice with $l$ children: one child for each of its (l-1)-subpatterns. The key idea of our algorithm is to store in each node $P$ the maximum confidence score for every class that can be obtained in the sublattice with top $P$ (including P itself): $\max _{-} \operatorname{con} f_{P}[c]=\max \left(\operatorname{Pr}\left(c \mid P^{\prime}\right)\right): \forall P^{\prime} \subseteq$ $P$. These max_conf values are computed from the bottom up as algorithm progresses. Initially, $\max { }_{-} \operatorname{con} f_{\Phi}$ for the empty pattern is set to be the prior distribution of the class variable. In order to compute $\max \_\operatorname{con} f_{P}$ for pattern $P$, we first compute $\operatorname{con} f_{P}$ (line 2), the distribution of the class variable in group $P$ : $\operatorname{conf}_{P}[c]=\operatorname{Pr}(c \mid P)$. Then we use the max_conf values of $P$ 's direct children to compute max_conf_children $P$ (line 3) so that max_conf_children $_{P}[c]=\max \left(\operatorname{Pr}\left(c \mid P^{\prime}\right)\right): \forall P^{\prime} \subset P$. Finally, we compute $\max \_$con $f_{P}$ by taking the element-wise maximum of two arrays: $\operatorname{con}_{P}$ and $\max _{\text {cconf_children }}$ (line 4).

After assigning the max_conf value for pattern $P$, we want to check if $P$ forms an MPR. So for each class label $c$, we perform the MPR significance test to check if $P$ predicts $c$ significantly better than $\max _{-}$conf_children ${ }_{P}[c]$. If the test is positive, we add the rule $P \Rightarrow c$ to the set of MPRs (line 8 ).

Please note that in our pseudo-code, we do not explicitly build the lattice. Instead, we use a hash table tbl_max_conf (Figure 3, line 1) to provide direct access to the max_conf values, so that $t b l \_m a x_{-} \operatorname{conf}[h(P)]=\max \_c o n f_{P}$, where $h$ is a hash function. Also note that none of the functions (calculate_class_distribution, is_MPP and lossless_pruning) requires another scan on the data because we can collect the class specific counts during the same scan that counts the pattern. 


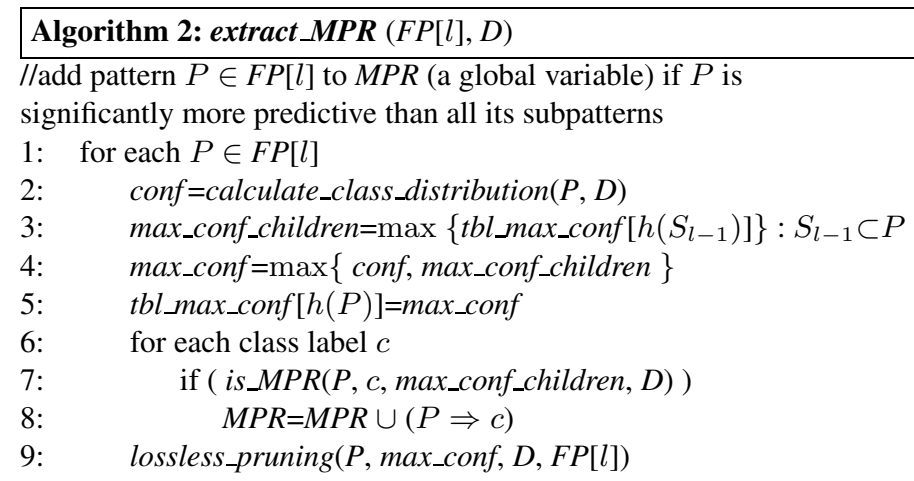

Function is_MPR(P, $c$, max_conf_children, $D)$

//return true if $P$ predicts $c$ significantly better than all its subpatterns

$N=\operatorname{count}(P, D)$

$N_{c}=\operatorname{count}(P \cup c, D)$

$p_{\text {_value }}=\operatorname{Pr}_{\text {binomial }}\left(x \geq N_{c} \mid N\right.$, max_conf_children $\left.[c]\right)$

if $\left(p_{-}\right.$value $\left.<\alpha\right)$

return true

return false

Function lossless_pruning $\left(P, \max _{-}\right.$conf, $\left.D, F P[l]\right)$

//prune $P$ if it cannot produce any $M P R$

for each class label $c$

$N_{c}=\operatorname{count}(P \cup c, D)$

$p_{-}$value $=P r_{\text {binomial }}\left(x \geq N_{c} \mid N_{c}, \max _{-}\right.$conf $\left.[c]\right)$

//exit if $P$ can potentially produce an $M P R$ for any class $c$

if $\left(p_{\text {_value }}<\alpha\right)$

return;

//Prune $P$

remove $(P, F P[l])$

Fig. 4. The algorithm for extracting MPRs from the frequent patterns at level $l$

Figure 5 illustrates the algorithm using a small lattice on a dataset that contains 200 instances from class $c_{1}$ and 300 instances from class $c_{2}$. For each pattern $P$ (node), we show the number of instances in each class, the distribution of the classes (conf) and the maximum confidence from $P$ 's sublattice (max_conf). Let us look for example at pattern $I_{1} \wedge I_{2}$. This pattern is predictive of class $c_{2}$ : conf $\left(I_{1} \wedge I_{2} \Rightarrow c_{2}\right)=0.75$. However, this rule is not an MPR since it does not significantly improve the predictability of $c_{2}$ over the subrule $I_{2} \Rightarrow c_{2}: P r_{\text {binomial }}(x \geq 75 \mid 100,0.7)=0.16$ (not significant at $\alpha=0.05)$. The MPRs from this example are: $I_{1} \Rightarrow c_{1}, I_{2} \Rightarrow c_{2}$ and $I_{1} \wedge I_{3} \Rightarrow c_{1}$.

Mining at low support. It is well know that the performance of the Apriori algorithm highly depends on the minimum support (min_sup) parameter. However, setting this parameter is not straightforward because the optimal min_sup can vary greatly across different datasets. In fact, [119] argued that it is sometimes of interest to mine low 


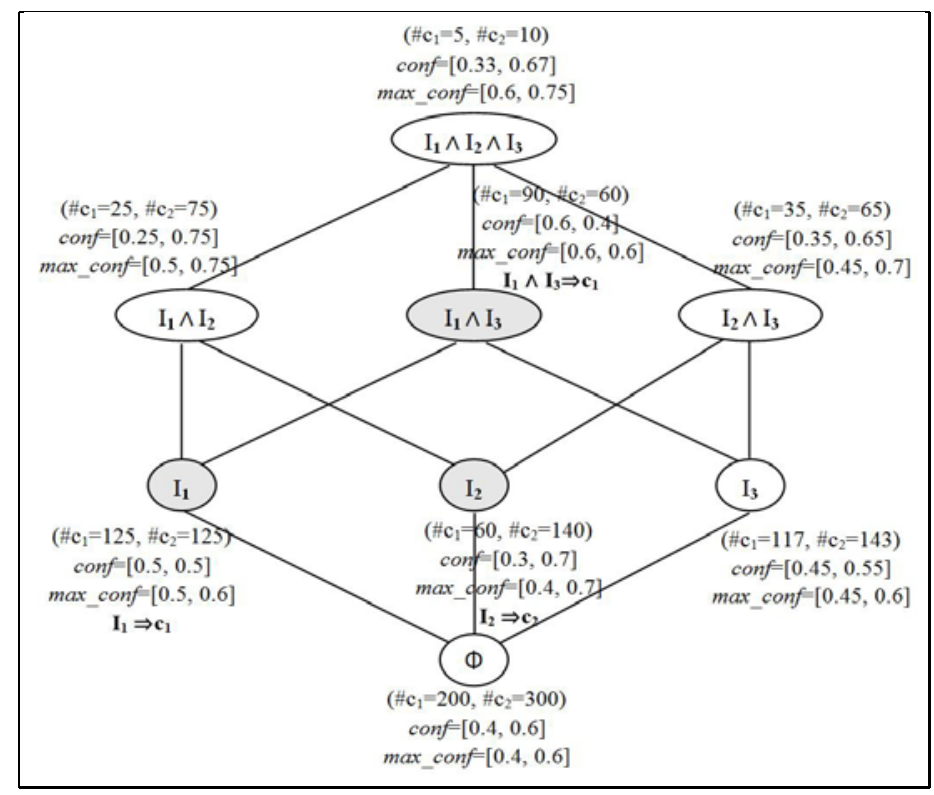

Fig. 5. An illustrative example showing the lattice associated with frequent pattern $I_{1} \wedge I_{2} \wedge I_{3}$. The MPRs are shaded.

support patterns. Therefore, in order not to miss any important pattern, the user may choose a low min_sup value. However, low min_sup raises two important concerns. First, the algorithm may return a huge number of rules, most which are very complex. Second, the algorithm may take very long time to finish.

In the following we argue that the MPR framework and our algorithm address both of these concerns. First, by requiring each MPR to be significantly better than all its subrules, the algorithm is biased towards choosing simple rules over more complex rules. Moreover, the MPR significance test incorporates the pattern's support because the chance of passing the test is lower for low support patterns. This acts as if the min_sup filter was built into the statistical significance test. Hence, very low support patterns are likely to be filtered out unless they are extremely predictive (with a very surprising distribution).

Second, the MPR significance test can help us to prune the search space. This early pruning is implemented by the lossless_pruning function in Figure 4 . The idea is that we can prune pattern $P$ if we guarantee that $P$ cannot produce any MPR. However, because the pruning is applied while generating the patterns, we do not know what subgroups $P$ will generate further in the lattice. To overcome this, let us define the optimal subgroup $P_{c_{i}}^{*}$ in group $P$ with respect to class $c_{i}$ to be the subgroup that contains all the instances from $c_{i}$ and none of the instances from any other classes. Clearly, $P$ cannot generate any subgroup better than $P_{c_{i}}^{*}$ for predicting class $c_{i}$. Now, we prune $P$ if for every class $c_{i}, P_{c_{i}}^{*}$ is not significant with respect to the best confidence so far max_conf $\left[c_{i}\right]$. Please note that this pruning technique does not miss any MPR because the lossless_pruning test is anti-monotonic. 
As an example, consider pattern $P=I_{1} \wedge I_{2} \wedge I_{3}$ in Figure 5. This pattern contains 15 examples, 5 from class $c_{1}$ and 10 from class $c_{2}$. Both $P_{c_{1}}^{*}$ and $P_{c_{2}}^{*}$ are not significant with respect to the current best predictions 0.6 and 0.75 (respectively). Therefore, there is no need to further explore $P$ 's subgroups and the entire sublattice can be safely pruned.

Correcting for multiple testing. When multiple rules are tested in parallel for significance, it is possible to learn a number of false rules by chance alone. This is a common problem for all techniques that rely on statistical tests. For example, assume we have 10,000 random items (independent of the class variable). If we test the significance of each of them with a significance level $\alpha=0.05$, then we expect about 500 items to be significant just by chance!

One approach to deal with the multiple hypothesis testing problem is to adjust the significance level at which each rule is tested. The most common way is the Bonferroni correction [25], which divides the significance level $(\alpha)$ by the number of tests performed. This approach is not suitable for rule mining because the number of rules tested is usually very large, resulting in an extremely low $\alpha$ and hence very few rules discovered. The other more recent approach, directly controls the false discovery rate (FDR) [7]: the expectation of the proportion of false discoveries in the result. We adopt the FDR correction method because it is less stringent and more powerful (has a lower Type II error) than the Bonferroni correction. We apply FDR as a post-processing step (Figure 3 line 10). It takes as input all potential MPRs with their p-values and outputs a subset of MPRs that satisfy the FDR criteria.

\section{Experiments}

In this section we present our experimental evaluation, first on synthetic datasets with known underlying patterns, and after that on several UCI classification datasets [2]. The experiments compare the performance of MPR against the following methods:

- complete: The set of all rules that cover more than min_sup examples in the data. We filter out useless rules by only including the ones that positively predict the class label (with a lift score [15] higher than one).

- closed: A subset of complete that corresponds to non-redundant rules based on the concept of closed frequent patterns [3].

- corr_chi: A subset of complete that contains the rules with significant positive correlations between the condition and conclusion according to the $\chi^{2}$ test [8|24|21].

- priorbinom: A subset of complete that contains the rules that are significantly different from the prior according to the binomial statistical test (section 2.3).

- prior_FDR: A subset of prior_binom that is post-processed using the false discovery rate (FDR) technique [7] to correct for the multiple testing problem.

- conf_imp: A subset of complete that satisfies the confidence improvement constraint [6 18 17]: each rule should have a higher confidence than all its subrules.

For all methods, we set the minimum support threshold (min_sup) to $10 \%$ the number of records in the dataset (unless otherwise stated). For the methods that use a statistical test: $c o r r \_c h i$, prior_binom, prior_FDR and $M P R$, we use the conventional significance level $\alpha=0.05$. 


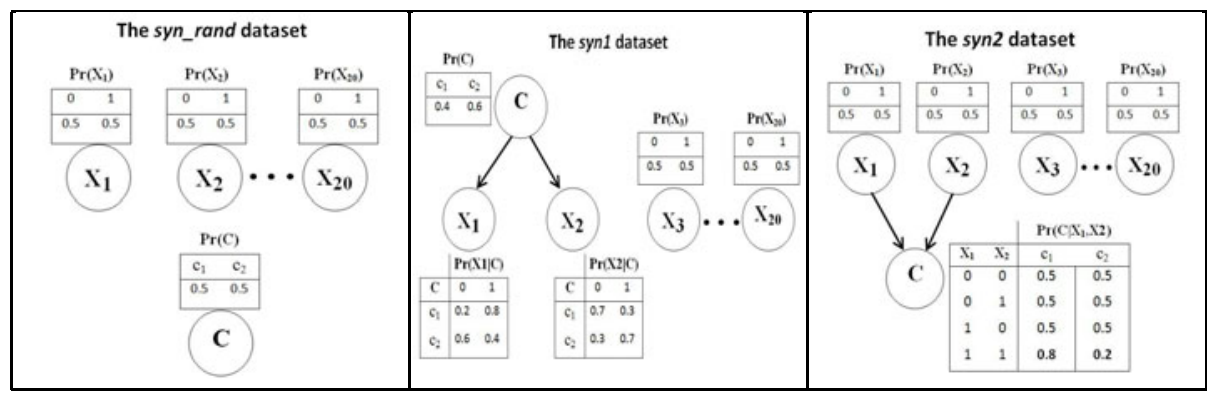

Fig. 6. Three Bayesian belief networks used to generate synthetic datasets: syn_rand, synl and syn2 (from left to right)

\subsection{Experiments on Synthetic Data}

The experiments on synthetic data (generated from pre-defined patterns) allow us to judge more objectively the quality of the algorithms' outputs by comparing the original and recovered patterns.

Data description. The synthetic data were generated from the three Bayesian belief networks (BBNs) in Figure 6 Each network consists of 20 random binary variables $\left\{X_{1}, \ldots, X_{20}\right\}$ and two class labels $c_{1}$ and $c_{2}$. The three networks are:

- syn_rand: In this network, all the attribute variables $X_{i}$ and the class variable $C$ are independent (syn_rand does not contain any pattern).

- syn1: In this network, $X_{1}=1$ is more frequent in class $c_{1}: \operatorname{Pr}\left(X_{1}=1 \mid C=c_{1}\right)=0.8$ $>\operatorname{Pr}\left(X_{1}=1 \mid C=c_{2}\right)=0.4$ and item $X_{2}=1$ is more frequent in class $c_{2}: \operatorname{Pr}\left(X_{2}=1 \mid\right.$ $\left.C=c_{2}\right)=0.7>\operatorname{Pr}\left(X_{2}=1 \mid C=c_{1}\right)=0.3$. Besides, the distribution of the class variable $C$ is more biased towards $c_{2}$ : $\operatorname{Pr}\left(C=c_{2}\right)=0.6$. The attributes $\left\{X_{3}, \ldots, X_{20}\right\}$ are independent of each other and the class variable $C$.

- syn2: The main pattern in syn 2 is a conjunction of $X_{1}=1$ and $X_{2}=1$ that predicts class $c_{1}: \operatorname{Pr}\left(C=c_{1} \mid X_{1}=1\right.$ and $\left.X_{2}=1\right)=0.8$. For all other values of $X_{1}$ and $X_{2}$, the two classes $\left(c_{1}\right.$ and $\left.c_{2}\right)$ are equally likely. The attributes $\left\{X_{3}, \ldots, X_{20}\right\}$ are independent of each other and the class variable $C$.

The datasets we analyze consist of 1000 examples randomly generated from these three networks.

Results: Table 1 summarizes the number of rules that each method produces on the three synthetic datasets. First notice that the number of rules in complete and closed are the same for all these datasets (since very few correlations exist). Also notice that corr_chi and prior_binom have similar results. This shows that the choice of the statistical test does not matter much and that the real problem with these approaches is that they evaluate each rule separately without considering the nested structure of the rules.

Let us look first at the results on the syn_rand dataset, which we know does not contain any pattern. MPR does not output any rule which is what we expect given that 
Table 1. The number of rules for the different methods on the synthetic datasets

\begin{tabular}{|c|c|c|c|c|c|c|c|}
\hline Dataset & complete & closed & corr_chi & prior_binom & prior_FDR & conf_imp & MPR \\
\hline \hline syn_rand & 9,763 & 9,763 & 516 & 651 & 0 & 4,748 & $\mathbf{0}$ \\
\hline syn1 & 9,643 & 9,643 & 2,989 & 3,121 & 2,632 & 4,254 & $\mathbf{6}$ \\
\hline syn2 & 9,868 & 9,868 & 1,999 & 2,225 & 422 & 3,890 & $\mathbf{5}$ \\
\hline
\end{tabular}

the class is independent of all attributes. On the other hand, conf_imp returns a huge number of rules. In fact, conf_imp returns almost half the total number of associations. This result agrees with our analysis in section 2.4 prior_FDR correctly removes all false positives from prior_binom on this random data.

Now consider the syn 1 dataset. Our algorithm extracts the following 6 MPRs: $\{X 1=1$ $\Rightarrow c 1, X 1=0 \Rightarrow c 2, X 2=1 \Rightarrow c 2, X 2=0 \Rightarrow c 1, X 1=0 \wedge X 2=1 \Rightarrow c 2, X 1=1 \wedge X 2=0 \Rightarrow$ c1 11 . In comparison, all other methods extract a vast number of rules. For example, even by using the FDR correction, the number of rules is 2,632 rules!

Table 2. The syn 2 dataset: on the left is the set of all MPRs, in the middle is the top 5 prior_binom rules (out of 2,225 rules) and on the right is the top 5 conf_imp rules (out of 3,890 rules)

\begin{tabular}{|c|c|c|}
\hline MPR & prior_binom & conf_imp \\
\hline $\begin{array}{c}\mathrm{X} 1=1 \wedge \mathrm{X} 2=1 \Rightarrow \mathrm{c} 1 \\
{[\text { sup }=26.5 \%, \text { conf }=81.1 \%]}\end{array}$ & $\begin{array}{c}\mathrm{X} 1=1 \wedge \mathrm{X} 2=1 \Rightarrow \mathrm{c} 1 \\
{[\text { sup }=26.5 \%, \text { conf }=81.1 \%}\end{array}$ & $\begin{array}{c}\mathrm{X} 1=1 \wedge \mathrm{X} 2=1 \wedge \mathrm{X} 8=0 \Rightarrow \mathrm{c} 1 \\
{[\text { sup }=12.3 \%, \text { conf }=85.4 \%]}\end{array}$ \\
\hline $\begin{array}{c}\mathrm{X} 2=1 \Rightarrow \mathrm{c} 1 \\
{[\text { sup }=50.1 \%, \text { conf }=66.7 \%]}\end{array}$ & $\begin{array}{c}\mathrm{X} 1=1 \wedge \mathrm{X} 2=1 \wedge \mathrm{X} 14=0 \Rightarrow \mathrm{c} 1 \\
{[\text { sup }=14.5 \%, \text { conf }=84.8 \%]}\end{array}$ & $\begin{array}{c}\mathrm{X} 1=1 \wedge \mathrm{X} 2=1 \wedge \mathrm{X} 14=0 \Rightarrow \mathrm{c} 1 \\
{[\text { sup }=14.5 \%, \text { conf }=84.8 \%]}\end{array}$ \\
\hline $\begin{array}{c}\mathrm{X} 1=1 \Rightarrow \mathrm{c} 1 \\
{[\text { sup }=51.2 \%, \text { conf }=63.9 \%}\end{array}$ & $\begin{array}{c}\mathrm{X} 1=1 \wedge \mathrm{X} 2=1 \wedge \mathrm{X} 13=1 \Rightarrow \mathrm{c} 1 \\
{[\text { sup }=13.4 \%, \text { conf }=84.3 \%]}\end{array}$ & $\begin{array}{c}\mathrm{X} 1=1 \wedge \mathrm{X} 2=1 \wedge \mathrm{X} 13=1 \Rightarrow \mathrm{c} 1 \\
{[\text { sup }=13.4 \%, \text { conf }=84.3 \%]}\end{array}$ \\
\hline $\begin{array}{c}\mathrm{X} 2=0 \Rightarrow \mathrm{c} 2 \\
{[\text { sup }=49.9 \%, \text { conf }=51.5 \%]}\end{array}$ & $\begin{array}{c}\mathrm{X} 1=1 \wedge \mathrm{X} 2=1 \wedge \mathrm{X} 9=1 \Rightarrow \mathrm{c} 1 \\
{[\text { sup }=14.1 \%, \text { conf }=83.7 \%]}\end{array}$ & $\begin{array}{c}\mathrm{X} 1=1 \wedge \mathrm{X} 2=1 \wedge \mathrm{X} 9=1 \Rightarrow \mathrm{c} 1 \\
{[\text { sup }=14.1 \%, \text { conf }=83.7 \%]}\end{array}$ \\
\hline $\begin{array}{c}\mathrm{X} 1=0 \Rightarrow \mathrm{c} 2 \\
{[\text { sup }=48.8 \%, \text { conf }=49.0 \%]}\end{array}$ & $\begin{array}{c}\mathrm{X} 1=1 \wedge \mathrm{X} 2=1 \wedge \mathrm{X} 8=0 \Rightarrow \mathrm{c} 1 \\
{[\text { sup }=12.3 \%, \text { conf }=85.4 \%]}\end{array}$ & $\begin{array}{c}\mathrm{X} 1=1 \wedge \mathrm{X} 2=1 \wedge \mathrm{X} 18=0 \Rightarrow \mathrm{c} 1 \\
{[\text { sup }=13.9 \%, \text { conf }=83.5 \%]}\end{array}$ \\
\hline
\end{tabular}

Finally, let us look more closely at the results on the syn 2 dataset. Table 2 shows all MPRs (left), the top 5 ranked rules for prior_binom (same for prior_FDR) according to the p-values (center) and the top 5 ranked rules for conf_imp according to the confidence (right). Notice that prior_binom correctly ranks the real pattern $\left(X_{1}=1 \wedge X_{2}=1\right)$ at the top. However, the following rules are redundant. For conf_imp, the real pattern is buried inside many spurious rules. This example clearly shows the deficiencies of these methods in concisely representing the actual patterns. On the contrary, by investigating the small number of MPRs, we can easily recover the structure of the underlying BBN.

\subsection{Experiments on UCI Datasets}

Data description. To further test the different methods, we use 9 public datasets from the UCI Machine Learning repository [2]. We discretize the numeric attributes into

\footnotetext{
${ }^{1}$ The last 2 rules combine the evidence of the two main patterns, hence improve the predictability. For example, $\operatorname{Pr}(\mathrm{c} 1 \mid \mathrm{x} 1=1 \wedge \mathrm{x} 2=0)>\operatorname{Pr}(\mathrm{c} 1 \mid \mathrm{x} 1=1)$.
} 
Table 3. UCI Datasets characteristics

\begin{tabular}{|c|c|c|c|c|}
\hline dataset & \# attributes & $\#$ items & \# records & \# classes \\
\hline \hline Adults & 14 & 154 & 32,561 & 2 \\
\hline Heart disease & 13 & 33 & 303 & 2 \\
\hline Lymphography & 18 & 57 & 142 & 2 \\
\hline Pima diabetes & 8 & 19 & 768 & 2 \\
\hline Breast cancer & 9 & 41 & 286 & 2 \\
\hline Dermatology & 12 & 47 & 366 & 6 \\
\hline Wine & 13 & 39 & 178 & 3 \\
\hline Glass & 10 & 22 & 214 & 2 \\
\hline Credit & 15 & 69 & 690 & 2 \\
\hline
\end{tabular}

Table 4. The number of rules for the different algorithms on several UCI datasets

\begin{tabular}{|c|c|c|c|c|c|c|c|}
\hline Dataset & complete & closed & corr_chi & prior_binom & prior_FDR & conf_imp & MPR \\
\hline \hline Adults & 2,710 & 2,042 & 2,616 & 2,620 & 2,619 & 374 & $\mathbf{1 5 2}$ \\
\hline Heart disease & 5,475 & 5,075 & 4,820 & 4,825 & 4,784 & 1,021 & $\mathbf{7 9}$ \\
\hline Lymphography & 31,594 & 5,840 & 15,740 & 15,032 & 11,627 & 978 & $\mathbf{2 4}$ \\
\hline Pima diabetes & 466 & 448 & 345 & 350 & 337 & 144 & $\mathbf{3 6}$ \\
\hline Breast cancer & 420 & 379 & 122 & 124 & 44 & 158 & $\mathbf{1 0}$ \\
\hline Dermatology & 5,350 & 3,727 & 3,820 & 3,717 & 3,544 & 2,076 & $\mathbf{9 6}$ \\
\hline Wine & 1,140 & 1,057 & 975 & 971 & 968 & 520 & $\mathbf{1 1 6}$ \\
\hline Glass & 2,327 & 1,141 & 2,318 & 2,311 & 2,311 & 97 & $\mathbf{2 0}$ \\
\hline Credit & 8,504 & 3,271 & 6,885 & 6,964 & 6,839 & 926 & $\mathbf{4 9}$ \\
\hline \hline Average & 6,443 & 2,553 & 4,182 & 4,102 & 3,675 & 699 & $\mathbf{6 5}$ \\
\hline
\end{tabular}

intervals by minimizing the entropy based on the minimum description length principle [14] (supervised discretization). Table 3 shows the main characteristics of the datasets. The number of items in column 3 is the number of all distinct attribute value pairs.

Results. Table 4 shows the number of rules for each method on the 9 UCI datasets. First notice that evaluating the rules individually based on their statistical significance (corr_chi, prior_binom and prior_FDR) does not help much in reducing the number of rules (even by using the FDR correction). It is clear from the table that the number of MPRs is much smaller than the number of rules in the other approaches. On average, MPRs are about two orders of magnitude smaller than complete and about one order of magnitude smaller than conf_imp.

Now we need a way to check if this small set of MPRs can adequately describe the datasets. However, we do not know what are the real patterns for these datasets. Hence, to evaluate the usefulness of the rules, we use the classification accuracy of the corresponding rule based classifier. We define two simple classifiers:

- Weighted confidence classification (w_conf): To classify instance $x$, we weight each rule that satisfy $x$ by its confidence and we choose the class that maximizes this weighted sum:

$$
w_{-} \operatorname{conf}(x)=\arg \max _{c_{i}}\left\{\sum_{x \subseteq A} \operatorname{conf}\left(A \Rightarrow c_{i}\right)\right\}
$$


- Highest confidence classification (h_conf): We classify instance $x$ according to the highest confidence rule that satisfy $x$ (this method is used in [20]):

$$
h_{-} \operatorname{conf}(x)=\arg \max _{c_{i}}\left\{\max _{x \subseteq A} \operatorname{conf}\left(A \Rightarrow c_{i}\right)\right\}
$$

We compare the classification accuracies obtained by using all association rules, using conf_imp rules (this approach was used in [17] for classification), and using MPR\$2. All of the reported results are obtained using 5-folds cross validation. Remember that the lift score for all rules is bigger than one (the condition and consequent of each rule are positively correlated). Hence, $w_{-}$conf consults only predictive rules.

Table 5. The classification accuracies (\%) for complete, conf_imp and MPR using two rule classification techniques: weighted confidence classification ( $w_{-}$conf $)$and highest confidence classification (h_conf)

\begin{tabular}{|c|c|c|c|c|c|c|}
\hline & \multicolumn{2}{|c|}{ complete } & \multicolumn{2}{c|}{ conf_imp } & \multicolumn{2}{c|}{ MPR } \\
Dataset & w_conf & h_conf & w_conf & h_conf & w_conf & h_conf \\
\hline \hline Adults & 77.3 & 75.9 & 80.6 & 75.9 & 80.8 & 75.9 \\
\hline Heart disease & 80.9 & 80.5 & 80.2 & 80.5 & 82.2 & 81.5 \\
\hline Lymphography & 81.2 & 83.6 & 71.8 & 83.6 & 86.2 & 85.9 \\
\hline Pima diabetes & 71.4 & 74.4 & 72.8 & 74.4 & 71.8 & 72.9 \\
\hline Breast cancer & 73.8 & 72.0 & 74.1 & 72.0 & 72.4 & 73.4 \\
\hline Dermatology & 68.0 & 69.4 & 58.2 & 69.4 & 63.4 & 65.6 \\
\hline Wine & 88.8 & 93.3 & 86.4 & 93.3 & 88.2 & 92.8 \\
\hline Glass & 94.4 & 100 & 93.5 & 100 & 95.8 & 100 \\
\hline Credit & 80.4 & 85.4 & 76.7 & 85.4 & 77.8 & 85.4 \\
\hline \hline Average & 79.6 & 81.6 & 77.1 & 81.6 & 79.8 & 81.5 \\
\hline
\end{tabular}

From Table 5, we can see that MPR does not sacrifice the classification accuracy. On average, all approaches produce comparable results for both $w_{-}$conf and $h_{-}$conf. An important benefit of using the compact set of MPRs for classification is that the classification time is very fast. For example, consider the Lymphography dataset. Instead of consulting 31,594 rules to classify each instance, MPR summarizes the classifier in only 24 rules. It is interesting to see that these 24 rules outperform the complete set of rules for both $w_{-}$conf and $h_{-}$conf.

Please note that we are not claiming that our approach can outperform the state-ofthe-art frequent pattern-based classifiers [13,10]. Our objective is just to show that even though MPRs provide a huge compression of the association rules, they can still capture the essential underlaying patterns in the data by providing comparable classification performance to using all association rules.

Mining at low support. In this section, we study the performance and the output of the different methods under different support thresholds. Due to the space limitation, we only show the results on the Heart disease dataset.

\footnotetext{
${ }^{2}$ corr_chi, prior_binom and prior_FDR gave similar classification results as complete, hence we excluded them from this table to save space.
} 


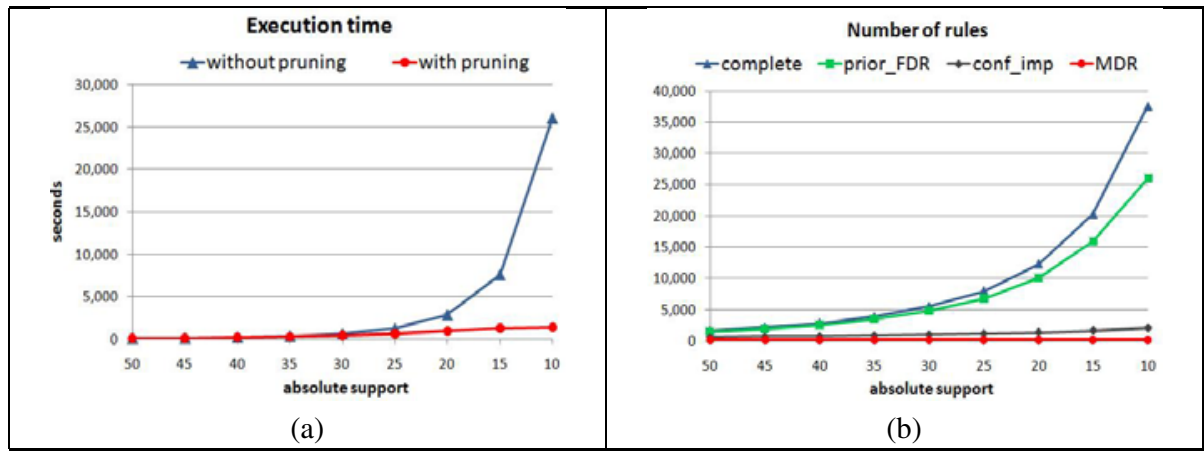

Fig. 7. The execution times (a) and the number of rules (b) of the algorithms on the heart disease dataset for different support thresholds

Figure 7 a shows the execution times using a Dell Precision T7500 machine with an Intel Xeon $3 \mathrm{GHz}$ CPU and 16GB of RAM. All algorithms are implemented in matlab. The "without pruning" chart corresponds to the execution of the Apriori algorithm that relies only on the support of the patterns to prune the search space. The "with pruning" chart corresponds to the execution of the algorithm that applies the additional MPR pruning technique in section 2.5 .

We can see that the execution time of Apriori exponentially blows up for low support values. On the other hand, the MPR pruning controls the complexity and the execution time increases very slowly for low support values. For example, when the absolute support threshold is 15 , which corresponds to min_sup $=5 \%$ on this dataset, applying the MPR pruning makes the algorithm about 6 times faster.

Figure $7 \mathrm{~b}$ shows the number of rules generated by the different methods. To improve the visibility, we did not include closed,prior_binom and corr_chi in this graph. We can see that the output of MPR does not change much when min_sup is very low. For example, by changing min_sup from 10\% (absolute support $=30$ ) to $5 \%$ (absolute support=15), the number of MPRs increases from 79 to 83 rules. In comparison, the same change causes the number of all association rules to increase from 5,475 to about 20,000 rules! Clearly, this large number of rules is overwhelming the user.

To summarize, our framework relieves the user from the burden of deciding the optimal min_sup by allowing him to conservatively set the support very low without drastically affecting the performance or the results of the algorithm.

\section{Related Research}

Several research attempts have been made to reduce the large number of association rules in order to make the results more suitable for knowledge discovery. Constrained associations rules methods [22] allow the user to define his own constraints and retrieve the rules that match these constraints. An opposite approach [23] mines the rules that are most different from the user's expectations. Maximal frequent itemsets [19] is a 
lossy compression of the frequent itemsets and cannot be used to generate rules. The profile based approach [27] is another lossy compression method.

The work in [6] aimed to reduce the number of class association rules by defining the confidence improvement constraint. This constraint was adopted by [18 17]. As we showed in the analysis and experiments, this approach can still generate many redundant rules. [21] defined the concept of direction setting (DS) rules in order to make browsing the class association rules easier for the user. However, their objective is different from ours because non-DS rules can indeed be significant MPRs. [5] extends the problem of association rule mining to the problem of mining contrasting sets. [26] defines a measure to rank the patterns by predicting the support of a pattern from the support of its subpatterns and measuring the deviation between the actual support and the prediction.

\section{Conclusion}

In this paper, we have developed a new framework for mining association rules based on the minimal predictive rules (MPR) concept. We showed that our method can produce a small set of predictive rules. Most importantly, each rule in the result is important because it concisely describes a distinct pattern that cannot be explained by any other rule in the set.

Motivated by our results, we plan to investigate the benefits of MPR for classification. In particular, we plan on incorporating the MPRs as additional features with the SVM classifier and comparing it against the state-of-the-art classifiers [13 10]. In addition, we plan to apply our method for anomaly detection in categorical data [12].

Acknowledgment. This research work was supported by grants 1R21LM009102-01A1, 1R01LM010019-01A1, and 1R01GM088224-01 from the NIH. Its content is solely the responsibility of the authors and does not necessarily represent the official views of the NIH.

\section{References}

1. Agrawal, R., Imielinski, T., Swami, A.: Mining association rules between sets of items in large databases. In: Proceedings of SIGMOD, pp. 207-216 (1993)

2. Asuncion, A., Newman, D.J.: UCI machine learning repository (2007)

3. Bastide, Y., Pasquier, N., Taouil, R., Stumme, G., Lakhal, L.: Mining minimal non-redundant association rules using frequent closed itemsets. In: Palamidessi, C., Moniz Pereira, L., Lloyd, J.W., Dahl, V., Furbach, U., Kerber, M., Lau, K.-K., Sagiv, Y., Stuckey, P.J. (eds.) CL 2000. LNCS (LNAI), vol. 1861, p. 972. Springer, Heidelberg (2000)

4. Batal, I., Sacchi, L., Bellazzi, R., Hauskrecht, M.: Multivariate time series classification with temporal abstractions. In: FLAIRS (2009)

5. Bay, S., Pazzani, M.: Detecting group differences: Mining contrast sets. Data Mining and Knowledge Discovery 5(3), 213-246 (2001)

6. Bayardo, R.J., Agrawal, R., Gunopulos, D.: Constraint-based rule mining in large, dense databases. In: Proceedings of ICDE, pp. 188-197 (1999)

7. Benjamini, Y., Hochberg, Y.: Controlling the false discovery rate: A practical and powerful approach to multiple testing. Journal of the Royal Statistical Society. Series B (Methodological) 57(1), 289-300 (1995) 
8. Brin, S., Motwani, R., Silverstein, C.: Beyond market baskets: Generalizing association rules to correlations. In: Proceedings of SIGMOD (1997)

9. Castelo, R., Feelders, A.J., Siebes, A.: Mambo: Discovering association rules based on conditional independencies. In: Hoffmann, F., Adams, N., Fisher, D., Guimarães, G., Hand, D.J. (eds.) IDA 2001. LNCS, vol. 2189, p. 289. Springer, Heidelberg (2001)

10. Cheng, H., Yan, X., Han, J., Hsu, C.: Discriminative frequent pattern analysis for effective classification. In: Proceedings of ICDE (2007)

11. Cohen, E., Datar, M., Fujiwara, S., Gionis, A., Indyk, P., Motwani, R., Ullman, J.D., Yang, C.: Finding interesting associations without support pruning. In: Proceedings of ICDE (2000)

12. Das, K., Schneider, J., Neill, D.: Anomaly pattern detection in categorical datasets. In: Proceedings of SIGKDD (2008)

13. Fan, W., Zhang, K., Cheng, H., Gao, J., Yan, X., Han, J., Yu, P., Verscheure, O.: Direct mining of discriminative and essential frequent patterns via model-based search tree. In: Proceedings of SIGKDD (2008)

14. Fayyad, U., Irani, K.: Multi-interval discretization of continuous-valued attributes for classification learning. In: Proceedings of IJCAI (1993)

15. Geng, L., Hamilton, H.: Interestingness measures for data mining: A survey. ACM Comput. Surv. 38(3) (2006)

16. Kuramochi, M., Karypis, G.: Frequent subgraph discovery. In: Proceedings of ICDM (2001)

17. Li, J., Shen, H., Topor, R.: Mining optimal class association rule set. In: Cheung, D., Williams, G.J., Li, Q. (eds.) PAKDD 2001. LNCS (LNAI), vol. 2035, p. 364. Springer, Heidelberg (2001)

18. Li, W., Han, J., Pei, J.: CMAR: Accurate and efficient classification based on multiple classassociation rules. In: Proceedings of ICDM (2001)

19. Lin, D., Kedem, Z.: Pincer-search: A new algorithm for discovering the maximum frequent set. In: Proceedings of EDBT, pp. 105-119 (1997)

20. Liu, B., Hsu, W., Ma, Y.: Integrating classification and association rule mining. In: Knowledge Discovery and Data Mining, pp. 80-86 (1998)

21. Liu, B., Hsu, W., Ma, Y.: Pruning and summarizing the discovered associations. In: Proceedings of SIGKDD (1999)

22. Ng, R., Lakshmanan, L., Han, J., Pang, A.: Exploratory mining and pruning optimizations of constrained associations rules. In: Proceedings of SIGMOD (1998)

23. Padmanabhan, B., Tuzhilin, A.: A belief-driven method for discovering unexpected patterns. In: Proceedings of SIGKDD (1998)

24. Piatetsky-Shapiro, G.: AAAI 1991 Workshop on Knowledge Discovery in Databases (1991)

25. Shaffer, J.P.: Multiple hypothesis testing: A review. Annual Review of Psychology (1995)

26. Tatti, N.: Maximum entropy based significance of itemsets. Knowledge Information System 17(1), 57-77 (2008)

27. Yan, X., Cheng, H., Han, J., Xin, D.: Summarizing itemset patterns: a profile-based approach. In: Proceedings of SIGKDD (2005)

28. Zaki, M.J.: Spade: an efficient algorithm for mining frequent sequences. Machine Learning Journal, 31-60 (2001) 